\title{
Comparison of full-endoscopic and minimally invasive decompression for lumbar spinal stenosis in the setting of degenerative scoliosis and spondylolisthesis
}

\author{
Saqib Hasan, MD, Lynn B. McGrath Jr., MD, Rajeev D. Sen, MD, Jason K. Barber, MS, and \\ Christoph P. Hofstetter, MD, PhD \\ Department of Neurological Surgery, University of Washington, Seattle, Washington
}

\begin{abstract}
OBJECTIVE The management of lumbar spinal stenosis (LSS) with concurrent scoliosis and/or spondylolisthesis remains controversial. Full-endoscopic unilateral laminotomy for bilateral decompression (ULBD) facilitates neural decompression while preserving stabilizing osseoligamentous structures and may be uniquely suited for the treatment of LSS with concurrent mild to moderate degenerative deformity. The safety and efficacy of full-endoscopic versus minimally invasive surgery (MIS) ULBD in this patient population is studied here for the first time.
\end{abstract}

METHODS A retrospective analysis of prospectively collected data was conducted on 45 consecutive LSS patients with concurrent scoliosis ( $\geq 10^{\circ}$ coronal Cobb angle) and/or spondylolisthesis $(\geq 3 \mathrm{~mm})$. Patient demographics, operative details, complications, and imaging characteristics were reviewed. Outcomes were quantified using back and leg visual analog scale (VAS) scores and the Oswestry Disability Index (ODI) at 2 weeks, 3 months, and 1 year.

RESULTS A total of 26 patients underwent full-endoscopic and 19 underwent MIS-ULBD with an average follow-up period of 12 months. The endoscopic cohort experienced a significantly shorter hospital length of stay $(p=0.014)$ and fewer adverse events $(p=0.010)$. Both cohorts experienced significant improvements in VAS and ODI scores at all time points $(p<0.001)$, but the endoscopic cohort demonstrated significantly better early ODI scores $(p=0.024)$.

CONCLUSIONS Endoscopic and MIS-ULBD result in similar functional outcomes for LSS with mild to moderate deformity, while the endoscopic approach demonstrates a favorable rate of complications. Further studies are required to better delineate the characteristics of spinal deformities amenable to this approach and the durability of functional results.

https://thejns.org/doi/abs/10.3171/2019.2.FOCUS195

KEYWORDS endoscopic spine surgery; stenosis; radiculopathy; minimally invasive spine surgery; MIS; scoliosis; unilateral laminotomy for bilateral decompression; ULBD

$\mathrm{L}$ UMBAR spinal stenosis (LSS) represents the most common indication for spine surgery in patients older than 65 years, with its prevalence expected to rise $59 \%$ to 64 million elderly adults by the year $2025.13,15,16,50$ While the treatment of patients with LSS without existing deformity or instability is primarily a decompressive procedure, there is a large subset of patients who have coexisting spondylolisthesis and/or degenerative deformity; the optimal treatment for these patients remains controversial.

The incidence and prevalence of degenerative scoliosis affecting adults has been reported variably, with curves greater than $10^{\circ}$ present in more than $50 \%$ of elderly females with back pain and osteoporosis and a new onset of deformity observed in over $30 \%$ of elderly patients. ${ }^{7,32,43}$ A population-based study using the National (Nationwide) Inpatient Sample database found that $82.7 \%$ of patients with LSS with coexisting spondylolisthesis and $67.6 \%$ of patients with coexisting scoliosis underwent a fusion procedure, while only $26.2 \%$ of patients with LSS without instability underwent a fusion procedure. ${ }^{4}$

Treatment options depend largely on patient factors and clinical presentation; patients with severe back pain and disability from significant sagittal or coronal imbalance are unlikely to benefit from a minimalist decompressive approach given the underlying structural problem. Patients whose symptoms are predominantly radiculopathy may be

ABBREVIATIONS ASA = American Society of Anesthesiologists; $L E$ = lumbar endoscopic; $L S S$ = lumbar spinal stenosis; $M C I D=$ minimally clinically important difference; MIS = minimally invasive surgery; ODI = Oswestry Disability Index; PI-LL = pelvic incidence-lumbar lordosis; ULBD = unilateral laminotomy for bilateral decompression; VAS = visual analog scale.

SUBMITTED January 1, 2019. ACCEPTED February 11, 2019.

INCLUDE WHEN CITING DOI: 10.3171/2019.2.FOCUS195. 
candidates for decompression-only procedures; however, even in this cohort, controversy remains, given early experiences with poor outcomes and curve progression following traditional open laminectomies. ${ }^{26,28}$

Minimally invasive decompressive techniques seek to minimize collateral damage and preserve the posterior elements with the purported advantage of preventing iatrogenic instability and curve progression associated with open techniques. , 22,52 While minimally invasive surgery using tubular-based unilateral laminotomy for bilateral decompression (MIS-ULBD) has been shown to be a clinically effective procedure in the treatment of a subset of LSS patients with degenerative scoliosis, ${ }^{23,37,38}$ there is a paucity of data on the effectiveness of the lumbar endoscopic ULBD (LE-ULBD) technique for this same cohort.

Endoscopic spine surgery represents the evolution of minimally invasive surgical access to spinal pathology. Multiple studies $27,34,40,45,49,56$ have demonstrated that endoscopic lumbar decompression in the setting of LSS provides equivalent outcomes to microsurgical or tubular techniques with shorter hospital stay and less collateral tissue injury. $2,40,41,47$ The purpose of this study was to evaluate and compare the clinical efficacy of LE-ULBD and MIS-ULBD decompression in patients with LSS and coexisting degenerative deformity.

\section{Methods \\ Patient Selection}

All participating patients provided written informed consent prior to undergoing the procedures as detailed. Collection of standard perioperative and postoperative outcome data are routinely performed as part of the University of Washington Spine Care Quality Initiative. Our prospectively collected database was retrospectively queried for ULBD performed with either a working channel endoscope (LE-ULBD) or an MIS technique using tubular retractors and the microscope (MIS-ULBD). ${ }^{11}$ Forty-five consecutive procedures were performed at the University of Washington between September 2014 and February 2017 by a single surgeon (C.P.H.). Conservative therapy in patients described in the current cohort, including at least 6 weeks of physical therapy, nonsteroidal antiinflammatory drugs, or epidural steroid injections, had failed. Only patients with LSS with coexisting stable degenerative spondylolisthesis (slip $\geq 3 \mathrm{~mm}$ ) or degenerative lumbar scoliosis $\left(\geq 10^{\circ}\right.$ coronal Cobb angle) were included in our study cohort. Patients were excluded if they had dynamic instability ( $>3 \mathrm{~mm}$ motion on flexion/extension radiographs), severe clinical sagittal imbalance (unable to stand upright), primary disc herniation, or a sequestered disc fragment.

Standard patient demographics, operative details, clinical outcomes, complications, and preoperative imaging measurements were recorded, including disc height, Cobb angle, static spondylolisthesis slip distance and grades, axial facet angle, pelvic incidence, and lumbar lordosis. Outcomes were quantified using visual analog scale (VAS) and Oswestry Disability Index (ODI) $\operatorname{scores}^{19}$ at 2 weeks, 3 months, and at 1 year. With respect to ODI scoring, we regarded the minimally clinically important dif- ference (MCID) $)^{8,25,29}$ to be 12 absolute points, and for VAS the MCID was 3 points. ${ }^{20,21,29}$

\section{Surgical Technique}

We used a previously described technique for MIS$\mathrm{ULBD}^{11}$ consisting of a traditional unilateral MIS approach through tubular retractors that can be used for bilateral decompression. The full-endoscopic ULBD technique has been previously described by the senior author. ${ }^{9}$ Briefly, sequential dilators are used to create a tubular path in which a beveled working cannula is placed, followed by a working-channel spinal endoscope (iLESSYS Delta, Joimax, Vertebris stenosis, RIWOSpine). Under direct endoscopic visualization, a high-speed diamond burr is used to create an ipsilateral laminotomy extending to the base of the spinous process, exposing the ligamentum flavum. The ligamentum flavum is then resected piecemeal using micropunches and Kerrison rongeurs to enter the epidural space. The contralateral lamina and medial facet are then undercut using the burr, and the ligamentum flavum is excised to decompress the contralateral traversing nerve root. The ipsilateral traversing nerve root is then decompressed by rotating the optics of the endoscope, allowing full visualization and access for medial facetectomy and resection of the ligamentum flavum. Patients in both cohorts were placed under general anesthesia and were permitted to leave the hospital the day of surgery; however, patients with medical comorbidities requiring close postoperative monitoring and patients older than 70 years were admitted to the hospital for observation.

\section{Statistical Analysis}

Continuous variables are presented as the mean \pm SD. Independent continuous variables were compared using a t-test. Categorical variables were compared using the chisquare test; $p<0.05$ was considered significant. Statistical calculations were carried out using IBM SPSS version 24 for Mac (IBM Corp.).

\section{Results}

The current study includes 45 patients with LSS with an average follow-up period of 12 months. Final followup data were available for $87 \%$ of our entire cohort. There were 19 patients who underwent MIS-ULBD and 26 patients who underwent LE-ULBD. Preoperative demographic and clinical characteristics, listed in Table 1 , demonstrated no significant differences between cohorts. Based on the American Society of Anesthesiologists (ASA) physical status classification system, 59\% of patients across cohorts were categorized as having severe systemic disease (ASA class III). Prior to surgery, patients in both cohorts were classified as severely disabled as indicated by a mean ODI score of $50.6 \pm 13.0$.

The decision to treat each patient with MIS or endoscopic technique was primarily related to equipment availability rather than surgical preference. All patients in the MIS cohort underwent surgery between September 2014 and June 2016, with the majority treated in 2015. After endoscopy equipment was adopted at the University of Washington, the endoscopic technique was performed al- 
TABLE 1. Patient demographics and preoperative clinical data

\begin{tabular}{|c|c|c|c|c|}
\hline & Overall & MIS-ULBD & LE-ULBD & $p$ Value \\
\hline No. of patients & 45 & 19 & 26 & \\
\hline Mean age, yrs (SD) & $68.5(10.3)$ & $66.6(8.0)$ & $69.9(11.6)$ & 0.171 \\
\hline \multicolumn{5}{|l|}{ Sex } \\
\hline Male & $24(53 \%)$ & $12(63 \%)$ & $12(46 \%)$ & \multirow{2}{*}{0.366} \\
\hline Female & $21(47 \%)$ & $7(37 \%)$ & $14(54 \%)$ & \\
\hline Mean BMI (SD) & $30.1(7.8)$ & $28.3(4.6)$ & $31.4(9.3)$ & 0.368 \\
\hline \multicolumn{5}{|l|}{ ASA class } \\
\hline 1 & $2(5 \%)$ & $0(0 \%)$ & $2(8 \%)$ & \multirow{4}{*}{0.934} \\
\hline II & $16(36 \%)$ & $8(42 \%)$ & $8(32 \%)$ & \\
\hline III & $26(59 \%)$ & $11(58 \%)$ & $15(60 \%)$ & \\
\hline IV & $0(0 \%)$ & $0(0 \%)$ & $0(0 \%)$ & \\
\hline Mean preop back VAS score (SD) & $6.3(2.7)$ & $7.1(2.2)$ & $5.8(2.9)$ & 0.187 \\
\hline Mean preop leg VAS score (SD) & $6.5(2.3)$ & $6.3(2.4)$ & $6.6(2.3)$ & 0.755 \\
\hline Mean preop ODI (SD) & $50.6(13.0)$ & $46.6(16.0)$ & $52.8(10.7)$ & 0.125 \\
\hline \multicolumn{5}{|l|}{ No. of levels } \\
\hline Mean (SD) & $1.7(0.8)$ & $1.8(0.9)$ & $1.6(0.7)$ & 0.790 \\
\hline Single & $20(44 \%)$ & $8(42 \%)$ & $12(46 \%)$ & $>0.99$ \\
\hline Multiple & $25(56 \%)$ & $11(58 \%)$ & $14(54 \%)$ & \\
\hline 2 & $21(84 \%)$ & $8(73 \%)$ & $13(93 \%)$ & 0.434 \\
\hline 3 & $2(8 \%)$ & $2(18 \%)$ & $0(0 \%)$ & \\
\hline 4 & $2(8 \%)$ & $1(9 \%)$ & $1(7 \%)$ & \\
\hline \multicolumn{5}{|l|}{ Surgical level (multiple possible) } \\
\hline $\mathrm{T} 12-\mathrm{L} 1$ & $1(2 \%)$ & $0(0 \%)$ & $1(4 \%)$ & $>0.99$ \\
\hline L1-2 & $4(9 \%)$ & $1(5 \%)$ & $3(12 \%)$ & 0.627 \\
\hline L2-3 & $13(29 \%)$ & $6(32 \%)$ & $7(27 \%)$ & 0.751 \\
\hline L3-4 & $23(51 \%)$ & $11(58 \%)$ & $12(46 \%)$ & 0.550 \\
\hline L4-5 & $28(62 \%)$ & $13(68 \%)$ & $15(58 \%)$ & 0.543 \\
\hline L5-S1 & $6(13 \%)$ & $1(5 \%)$ & $5(19 \%)$ & 0.222 \\
\hline \multicolumn{5}{|l|}{ Pathology } \\
\hline LSS only & $36(80 \%)$ & $16(84 \%)$ & $20(77 \%)$ & 0.407 \\
\hline LSS + disc herniation & $5(11 \%)$ & $3(16 \%)$ & $2(8 \%)$ & \\
\hline LSS + synovial cyst & $2(4 \%)$ & $0(0 \%)$ & $2(8 \%)$ & \\
\hline LSS + foraminal stenosis & $2(4 \%)$ & $0(0 \%)$ & $2(8 \%)$ & \\
\hline \multicolumn{5}{|l|}{ Presence of deformity } \\
\hline Spondylolisthesis only & $6(13 \%)$ & $2(11 \%)$ & $4(15 \%)$ & 0.158 \\
\hline Scoliosis only & $16(36 \%)$ & $4(21 \%)$ & $12(46 \%)$ & 0.877 \\
\hline Scoliosis w/ spondylolisthesis & $23(51 \%)$ & $13(68 \%)$ & $10(38 \%)$ & \\
\hline Mean PI-LL mismatch, ${ }^{\circ}$ (SD) & $15.6(11.5)$ & $14.4(9.4)$ & $16.6(13.3)$ & \\
\hline Mean EBL, mL (SD) & $17.3(16.7)$ & $30.0(18.9)$ & $3.1(5.0)$ & $<0.001$ \\
\hline Mean length of stay, days (SD) & $1.2(1.1)$ & $1.7(1.2)$ & $0.9(0.8)$ & 0.014 \\
\hline
\end{tabular}

$\mathrm{EBL}=$ estimated blood loss.

Values are presented as the number of patients (\%) unless stated otherwise. Boldface type indicates statistical significance by Mann-Whitney and Fisher's exact test as appropriate.

most exclusively from June 2016 until the endpoint of the study in February 2017. One important exception to this was a tendency to perform the MIS technique in the case of 3-level decompressions, which were rare.

Patient operative data are listed in Table 1. The total number of operative levels was 75 , with 32 operative levels in the MIS-ULBD cohort and 43 operative levels in the LE-ULBD cohort. Within the MIS-ULBD cohort, $42 \%$ of patients underwent 1-level surgery and 58\% of patients underwent multilevel surgery. Within the LE-ULBD cohort, $46 \%$ of patients underwent 1-level surgery and 54\% of patients underwent multilevel surgery. The majority of 
TABLE 2. Radiographic data by subgroup

\begin{tabular}{|c|c|c|c|c|}
\hline & Overall & MIS-ULBD & LE-ULBD & $\mathrm{p}$ Value \\
\hline \multicolumn{5}{|l|}{ Spondylolisthesis subgroup } \\
\hline Mean PI, ${ }^{\circ}(\mathrm{SD})$ & $59.5(12.2)$ & $58.0(14.8)$ & $60.6(10.1)$ & 0.373 \\
\hline Mean LL, ${ }^{\circ}(\mathrm{SD})$ & $49.0(10.1)$ & $51.0(11.2)$ & $46.9(8.9)$ & 0.306 \\
\hline Mean PI-LL mismatch, ${ }^{\circ}$ (SD) & $13.6(10.7)$ & $13.6(11.3)$ & $13.7(10.6)$ & 0.979 \\
\hline Mean disc height, mm (SD) & $9.2(3.2)$ & $9.2(3.3)$ & $9.3(3.1)$ & 0.594 \\
\hline Mean slip measurement, mm (SD) & $6.2(2.8)$ & $6.5(3.4)$ & $5.9(2.1)$ & 0.941 \\
\hline Mean axial facet angle, ${ }^{\circ}(\mathrm{SD})$ & $47.9(15.5)$ & $48.3(12.8)$ & $47.5(18.6)$ & 0.642 \\
\hline \multicolumn{5}{|l|}{ Scoliosis subgroup } \\
\hline Mean Cobb angle, ${ }^{\circ}(\mathrm{SD})$ & $15.9(7.6)$ & $15.6(6.0)$ & $16.1(8.7)$ & $>0.99$ \\
\hline Mean $\mathrm{PI},{ }^{\circ}(\mathrm{SD})$ & $60.0(12.3)$ & $62.5(14.9)$ & $58.5(10.6)$ & 0.674 \\
\hline Mean LL, ${ }^{\circ}(\mathrm{SD})$ & $46.9(11.1)$ & $49.7(10.9)$ & $44.9(11.0)$ & 0.171 \\
\hline Mean PI-LL mismatch, ${ }^{\circ}(\mathrm{SD})$ & $14.9(11.8)$ & $14.9(10.8)$ & $15.0(12.7)$ & 0.827 \\
\hline Mean disc height, mm (SD) & $9.0(3.0)$ & $9.6(2.7)$ & $8.7(3.1)$ & 0.204 \\
\hline Mean axial facet angle, ${ }^{\circ}(\mathrm{SD})$ & $46.4(15.4)$ & $48.8(12.4)$ & $44.7(17.2)$ & 0.580 \\
\hline \multicolumn{5}{|l|}{ Lateral listhesis, no. } \\
\hline None & $27(41 \%)$ & $12(44 \%)$ & $15(38 \%)$ & 0.610 \\
\hline Positive & $39(59 \%)$ & $15(56 \%)$ & $24(62 \%)$ & \\
\hline Mean, mm (SD) & $6.1(2.4)$ & $6.1(1.8)$ & $6.0(2.7)$ & 0.945 \\
\hline
\end{tabular}

Statistical significance by Mann-Whitney (patients) and mixed-effects rank-linear and logistic regression (surgery levels).

patients in both groups were treated for central and lateral recess stenosis; 9 patients were treated for additional pathology including nonsequestered disc herniation (11\%), synovial cyst (4\%), and foraminal stenosis (4\%). The most common surgical level was L4-5, with $62 \%$ of patients undergoing surgery at this level. Average estimated blood loss was significantly less in the LE-ULBD cohort than in the MIS-ULBD cohort $(\mathrm{p}<0.001)$. Average hospital length of stay was significantly greater in the MIS-ULBD cohort (1.7 \pm 1.2 days) than in the LE-ULBD cohort $(0.9 \pm$ 0.8 days) $(\mathrm{p}=0.014)$.

With regard to presence of deformity, $13 \%$ of our cohort had spondylolisthesis with no coexisting scoliosis, $36 \%$ of our cohort had scoliosis with no coexisting spondylolisthesis, and $51 \%$ had the presence of both spondylolisthesis and scoliosis. There were no significant differences in presence or degree of deformity across cohorts. Preoperative radiographic data are listed in Tables 2 and 3. Patients with scoliosis presented with an average Cobb angle of $15.9^{\circ} \pm 7.6^{\circ}$, and $59 \%$ of patients demonstrated lateral listhesis (mean $6.1 \pm 2.4 \mathrm{~mm}$ ). Patients with spondylolisthesis presented with an average slip of $6.2 \pm 2.8$ $\mathrm{mm}$. The average disc height was $9.2 \pm 3.2 \mathrm{~mm}$, and the average axial facet angle was $47.9^{\circ} \pm 15.5^{\circ}$. The average pelvic incidence-lumbar lordosis (PI-LL) mismatch for the entire cohort was $15.6^{\circ} \pm 11.5^{\circ}$.

Patient-reported outcomes at all follow-up intervals are reported in Tables 3 and 4. Patients in the total cohort experienced significant improvement in VAS back pain, VAS leg pain, and ODI when comparing preoperative values to all postoperative time points ( $p<0.001$, Fig. 1). When comparing outcomes between the MIS-ULBD and LE-ULBD, the endoscopic cohort demonstrated significantly better early ODI scores $(\mathrm{p}=0.024)$; however, there were no significant differences at later time points. The percentage of patients reaching MCID for VAS leg pain in the MIS-ULBD and LE-ULBD groups was $82 \%$ and $95 \%$, respectively (Table 5). The percentage of patients reaching MCID for ODI in the MID-ULBD group and LE-ULBD group was $86 \%$ for both groups. There was no significant difference in the percentage of patients reaching MCID for both outcome measures.

The number of total adverse events experienced can be found in Table 5. An adverse event was defined as any perioperative medical event (any medical event requiring medical consultation or delaying discharge), intraoperative complications such as incidental durotomy, infection, or any reoperation within the follow-up period. The total number of perioperative medical events for patients in the MIS-ULBD cohort was greater than those experienced in the LE-ULBD cohort (5 [26\%] vs 2 [8\%] events); however, this difference was not statistically significant. Perioperative medical complications included urinary retention $(\mathrm{n}=$ $4)$, syncope $(n=1)$, stroke $(n=1)$, and laboratory abnormalities requiring medical consultation $(\mathrm{n}=1)$. Two patients $(11 \%)$ in the MIS-ULBD group and 0 patients $(0 \%)$ in the LE-ULBD group sustained an incidental durotomy $(p=0.173)$. There were no infections in either group.

Two patients requiring reoperation, with a total reoperation rate of $4 \%$. The reoperation rate at 1 year for the MIS-ULBD cohort was $11 \%$ versus $0 \%$ in the LE-ULBD cohort, although this was not statistically significant $(\mathrm{p}$ $=0.173$ ). All reoperations in the MIS-ULBD cohort occurred at an average time to reoperation of 6 months. All patients who required reoperation had recurrent leg symptoms at the index level and were treated with endoscopic transforaminal decompression. When looking at all adverse events, including all perioperative complications and 


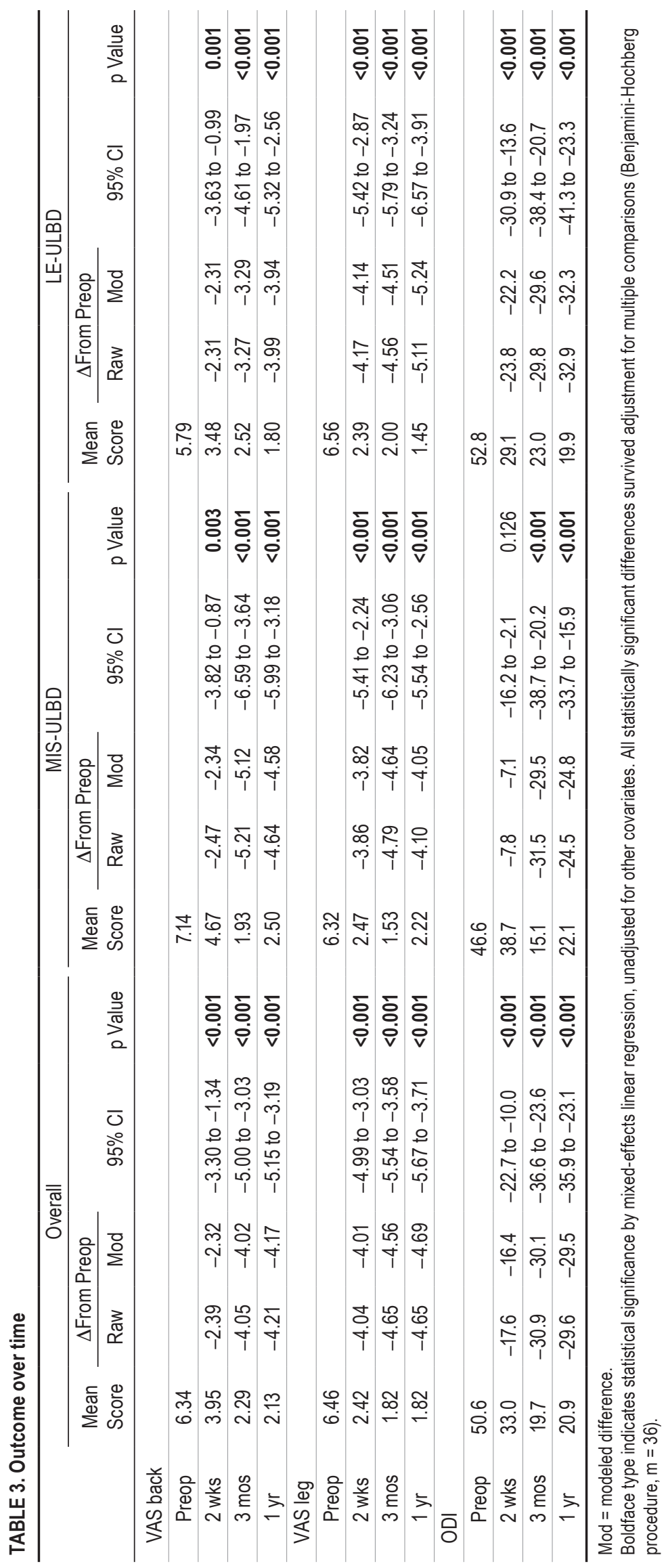


TABLE 4. Effects of surgery group on outcome (over time)

\begin{tabular}{|c|c|c|c|c|c|c|c|c|c|}
\hline & \multicolumn{2}{|c|}{ Score } & \multicolumn{3}{|c|}{$\Delta$ From Preop } & \multicolumn{4}{|c|}{ Model Estimates } \\
\hline & MIS-ULBD & LE-ULBD & MIS-ULBD & LE-ULBD & Difference & LE-ULBD Effect & $95 \% \mathrm{Cl}$ & $p$ Value & Favor \\
\hline \multicolumn{10}{|l|}{ VAS back } \\
\hline Preop & 7.14 & 5.79 & & & & & & & \\
\hline 2 wks & 4.67 & 3.48 & -2.47 & -2.31 & 0.16 & 0.00 & -1.99 to 2.00 & 0.997 & MIS-ULBD \\
\hline $3 \mathrm{mos}$ & 1.93 & 2.52 & -5.21 & -3.27 & 1.94 & 1.81 & -0.18 to 3.80 & 0.075 & MIS-ULBD \\
\hline $1 \mathrm{yr}$ & 2.50 & 1.80 & -4.64 & -3.99 & 0.65 & 0.63 & -1.35 to 2.60 & 0.531 & MIS-ULBD \\
\hline \multicolumn{10}{|l|}{ VAS leg } \\
\hline Preop & 6.32 & 6.56 & & & & & & & \\
\hline 2 wks & 2.47 & 2.39 & -3.86 & -4.17 & -0.31 & -0.32 & -2.33 to 1.69 & 0.754 & LE-ULBD \\
\hline $3 \mathrm{mos}$ & 1.53 & 2.00 & -4.79 & -4.56 & 0.23 & 0.14 & -1.88 to 2.15 & 0.894 & MIS-ULBD \\
\hline $1 \mathrm{yr}$ & 2.22 & 1.45 & -4.10 & -5.11 & -1.01 & -1.20 & -3.17 to 0.78 & 0.233 & LE-ULBD \\
\hline \multicolumn{10}{|l|}{ ODI } \\
\hline Preop & 46.6 & 52.8 & & & & & & & \\
\hline 2 wks & 38.7 & 29.1 & -7.8 & -23.8 & -15.9 & -15.0 & -28.0 to -2.0 & 0.024 & LE-ULBD \\
\hline 3 mos & 15.1 & 23.0 & -31.5 & -29.8 & 1.7 & 0.1 & -13.1 to 13.2 & 0.991 & MIS-ULBD \\
\hline $1 \mathrm{yr}$ & 22.1 & 19.9 & -24.5 & -32.9 & -8.5 & -7.5 & -20.5 to 5.4 & 0.252 & LE-ULBD \\
\hline
\end{tabular}

Boldface type indicates statistical significance by mixed-effects linear regression, unadjusted for other covariates. No statistically significant differences after adjusting for multple comparisons (Benjamini-Hochberg, $m=12$ ).

reoperations, the MIS-ULBD cohort was found to have a statistically significant greater number of adverse events $(\mathrm{n}=8)$ than the LE-ULBD cohort $(\mathrm{n}=2)(\mathrm{p}=0.010)$. No patient experienced postoperative iatrogenic motor or sensory deficits.

A poor outcome was defined as any reoperation or failure to reach MCID for leg VAS and ODI score. Univariate analyses were used to predict poor outcomes from preoperative radiographic data (Tables 6 and 7). There was a trend toward poor outcome with larger Cobb angles, larger disc heights, and more sagittally oriented facet angles; however, this was not statistically significant after adjusting for multiple comparisons. No other radiographic variables were found to be predictive of poor outcomes.

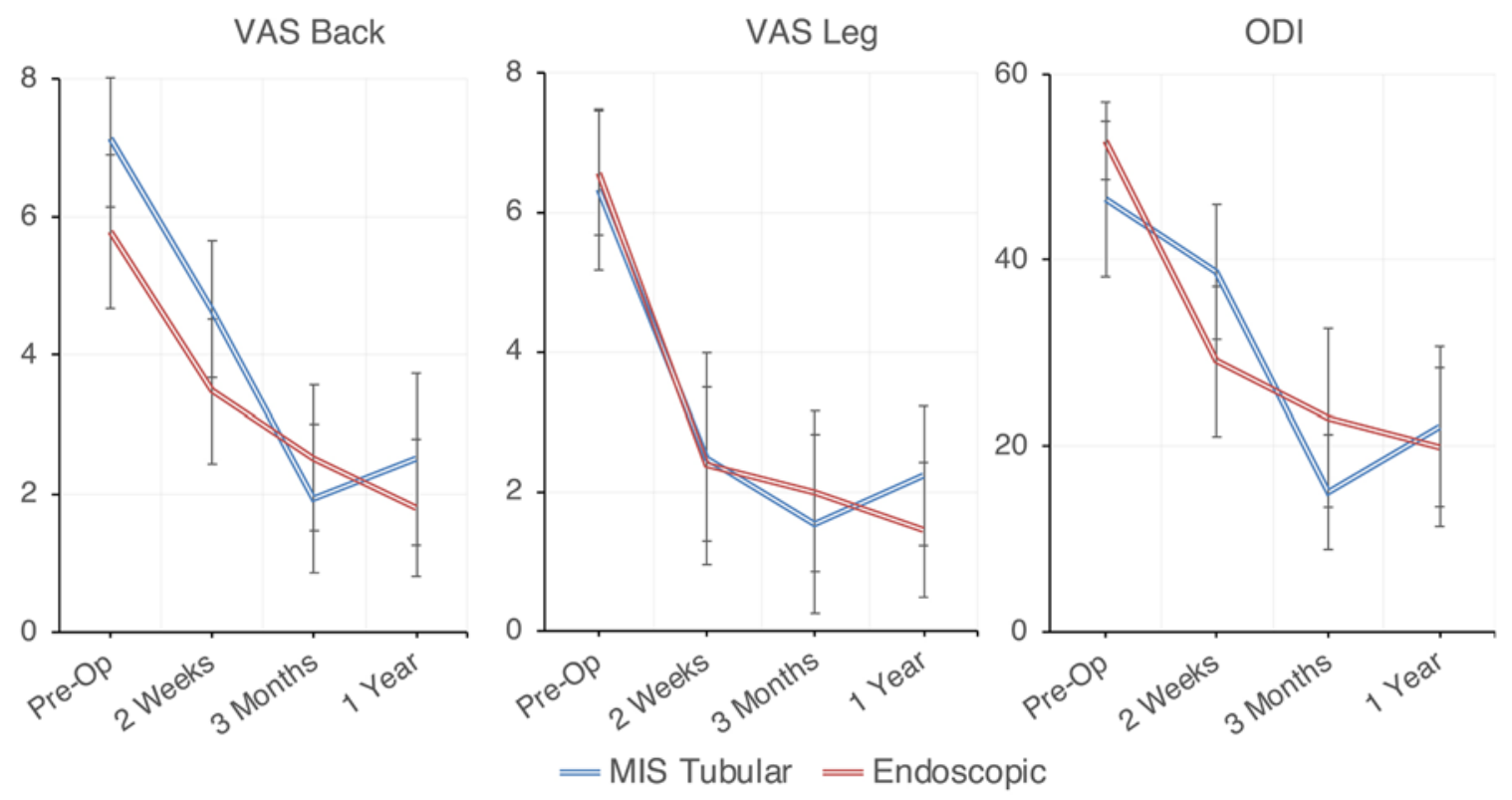

FIG. 1. Patients in the total cohort experienced significant improvement in VAS back pain, VAS leg pain, and ODI when comparing preoperative values to all postoperative time points $(p<0.001)$. When comparing outcomes between the MIS-ULBD and LE-ULBD cohorts, the endoscopic cohort demonstrated significantly better early ODI scores $(p=0.024)$; however, there were no significant differences at later time points. Means and SDs are shown. 
TABLE 5. Adverse events and MCID effects of surgery group on outcome (over time)

\begin{tabular}{|c|c|c|c|c|}
\hline & \multicolumn{3}{|c|}{ No. of Patients (\%) } & \multirow{2}{*}{$\begin{array}{c}p \\
\text { Value }\end{array}$} \\
\hline & Overall & MIS-ULBD & LE-ULBD & \\
\hline \multicolumn{5}{|l|}{ Adverse events } \\
\hline Any adverse event & $10(22)$ & $8(42)$ & $2(8)$ & 0.010 \\
\hline Periop medical event & $7(16)$ & $5(26)$ & $2(8)$ & 0.114 \\
\hline Intraop durotomy & $2(4)$ & $2(11)$ & $0(0)$ & 0.173 \\
\hline Infection & $0(0)$ & $0(0)$ & $0(0)$ & \\
\hline Reop & $2(4)$ & $2(11)$ & $0(0)$ & 0.173 \\
\hline \multicolumn{5}{|l|}{ MCID for VAS leg at $1 \mathrm{yr}$} \\
\hline No & $4(11)$ & $3(18)$ & $1(5)$ & \multirow{2}{*}{0.326} \\
\hline Yes & $32(89)$ & $14(82)$ & $18(95)$ & \\
\hline \multicolumn{5}{|l|}{ MCID for ODI at $1 \mathrm{yr}$} \\
\hline No & $5(14)$ & $2(14)$ & $3(14)$ & \multirow{2}{*}{$>0.99$} \\
\hline Yes & $30(86)$ & $12(86)$ & $18(86)$ & \\
\hline
\end{tabular}

Boldface type indicates statistical significance.

\section{Discussion}

Surgical decompression without fusion has been well accepted as the optimal treatment for patients with uncomplicated LSS causing neurogenic claudication. ${ }^{54}$ Clinical decision-making becomes more challenging when patients with LSS present with coexisting spondylolisthesis and/ or degenerative scoliosis. The ongoing debate regarding decompression alone versus decompression with fusion in this specific cohort has largely been informed by data pertaining to decompression via conventional midline laminectomy. In their landmark controlled trial, Herkowitz and Kurz demonstrated a high failure rate in patients with spondylolisthesis after a conventional midline muscle-stripping laminectomy. ${ }^{33}$ Consequently, this resulted in the widespread investigation of fusion with decompression for any patient with LSS and concomitant structural abnormalities. In the United States, $96 \%$ of patients with degenerative spondylolisthesis undergo fusion surgery as an adjunct to decompression, ${ }^{39}$ and approximately $70 \%$ of patients with LSS and coexisting scoliosis undergo a fusion procedure. ${ }^{4}$

Fusion itself, however, can be complicated by pseudarthrosis ${ }^{18}$ and adjacent-segment disease, ${ }^{44}$ ultimately leading to loss of therapeutic sustainability over time. A large analysis of registry data showed that the addition of fusion surgery to decompression surgery for spinal stenosis doubled the risk of severe adverse events. ${ }^{14,16}$ The potential risks and complications are significantly amplified when the alternative to decompression includes long hardware constructs, with some series showing complication rates greater than $50 \% .^{55}$

Based on the available literature, there is considerable variability in outcomes following decompression to address stenosis in the setting of degenerative scoliosis and spondylolisthesis. Several early studies have reported high rates of progressive deformity and failure of conventional laminectomy in patients with deformity and symptomatic stenosis. ${ }^{1,6,17,24,36,48}$ More recent literature regarding con-
TABLE 6. Univariate prediction of poor outcome from radiographic data

\begin{tabular}{lccc}
\hline & \multicolumn{3}{c}{ Poor Outcome $^{*}$} \\
\cline { 2 - 4 } & OR & $95 \% \mathrm{Cl}$ & $\mathrm{p} \mathrm{Value}$ \\
\hline Patient characteristics & & & \\
\hline Spondylolisthesis & 1.39 & $0.18-17.4$ & $>0.99$ \\
\hline Scoliosis & 0.60 & $0.06-8.55$ & 0.958 \\
\hline Cobb angle (per 5 ${ }^{\circ}$ ) & 0.67 & $0.36-1.16$ & 0.166 \\
\hline Level characteristics & & & \\
\hline Disc height (per mm) & 1.19 & $0.92-1.58$ & 0.186 \\
\hline Axial facet angle (per $5^{\circ}$ ) & 1.17 & $0.93-1.51$ & 0.188 \\
\hline Lateral listhesis & 0.56 & $0.11-2.70$ & 0.614 \\
\hline
\end{tabular}

Statistical significance by exact logistic regression. No statistically significant differences after adjusting for multiple comparisons (Benjamini-Hochberg, $m=6)$.

* Poor outcome defined as any of 1) reoperation, 2) no MCID for VAS leg, or 3) no MCID for ODI.

ventional laminectomy has painted a conflicting picture. One study found lower functional outcome scores and higher complication rates (56\% vs $10 \%)$ in patients undergoing large construct fusion with decompression than in patients receiving decompression alone; however, patients in the decompression group were less likely to respond favorably to questionnaires regarding patient satisfaction than those in the fusion groups.$^{53}$ Brodke et al. ${ }^{12}$ conducted a retrospective review comparing 3 treatment arms for radiculopathy in the setting of deformity. Ninety consecutive patients with a minimum 2-year follow-up were treated for LSS with associated deformity (mean Cobb angle $17^{\circ}$ ) with interspinous device placement, laminectomy alone, or laminectomy and short-segment fusion. The index-level reoperation rates were $33.3 \%, 8.3 \%$, and $0 \%$, respectively; however, the reoperation rate due to adjacent-segment disease was highest in the fusion group (24.4\%). The authors determined that conventional laminectomy alone had the highest rate of survival. One retrospective study found that in 50 patients who underwent traditional open decompression procedures in the setting of mild to moderate deformity with a mean follow-up 2.8 years, the average curve progression was $3.4^{\circ}$ with a mean reoperation rate of $10 \% .{ }^{35}$ These studies highlight the lack of certainty regarding the role of conventional laminectomy and potential iatrogenic instability in LSS patients with coexisting deformity, with reoperation rates ranging from $8 \%$ to $37 \%$. These results must also be taken into the context of the natural progression of degeneration that occurs with aging.

The advent of minimally invasive, midline-sparing techniques have revisited the validity of performing fusion in all patients who have a deformity associated with their symptomatic stenosis. Numerous biomechanical studies $^{30,31}$ have demonstrated that minimally invasive techniques help maintain spinal stability when compared with more conventional open techniques, presumably by sparing osseoligamentous structures. A recent study based on finite-element analysis found that endoscopic decompression has no negative impact on mechanical stability. ${ }^{46}$ These less-invasive techniques are particularly useful in 
TABLE 7. Univariate prediction of reoperation and inability to reach MCID from radiographic data

\begin{tabular}{|c|c|c|c|c|c|c|c|c|c|}
\hline & \multicolumn{3}{|c|}{ Reop } & \multicolumn{3}{|c|}{ No MCID for VAS Leg } & \multicolumn{3}{|c|}{ No MCID for ODI } \\
\hline & OR & $95 \% \mathrm{Cl}$ & $p$ Value & OR & $95 \% \mathrm{Cl}$ & $p$ Value & OR & $95 \% \mathrm{Cl}$ & p Value \\
\hline \multicolumn{10}{|l|}{ Patient characteristics } \\
\hline Spondylolisthesis & 1.36 & 0.16 to $\infty$ & 0.820 & 0.40 & 0.03 to 6.34 & 0.712 & 3.09 & 0.51 to $\infty$ & 0.327 \\
\hline Scoliosis & 0.37 & 0.04 to $\infty$ & $>0.99$ & 1.12 & 0.17 to $\infty$ & 0.931 & 0.18 & 0.01 to 2.95 & 0.278 \\
\hline Cobb angle (per $\left.5^{\circ}\right)$ & 0.75 & 0.26 to 1.76 & 0.582 & 0.91 & 0.46 to 1.68 & 0.801 & 0.52 & 0.23 to 1.02 & 0.058 \\
\hline PI-LL mismatch & 1.02 & 0.89 to 1.14 & 0.698 & 0.90 & 0.67 to 1.07 & 0.383 & 0.96 & 0.82 to 1.07 & 0.554 \\
\hline \multicolumn{10}{|l|}{ Level characteristics } \\
\hline Disc height (per mm) & 1.14 & 0.78 to 1.78 & 0.534 & 1.01 & 0.74 to 1.40 & $>0.99$ & 1.99 & 1.20 to 4.03 & 0.003 \\
\hline Slip (per mm) & 0.82 & 0.37 to 1.47 & 0.663 & 0.84 & 0.40 to 1.49 & 0.735 & 0.76 & 0.43 to 1.16 & 0.250 \\
\hline Axial facet angle $\left(\right.$ per $\left.5^{\circ}\right)$ & 1.61 & 0.96 to 3.17 & 0.078 & 0.89 & 0.67 to 1.19 & 0.405 & 1.35 & 0.95 to 2.09 & 0.108 \\
\hline Lateral listhesis & 3.50 & 0.52 to $\infty$ & 0.293 & 0.21 & 0.00 to 2.33 & 0.311 & 0.15 & 0.00 to 1.62 & 0.154 \\
\hline Lateral listhesis (per mm) & 0.95 & 0.57 to 1.59 & 0.852 & 0.83 & 0.22 to 2.11 & 0.733 & 0.35 & 0.01 to 1.29 & 0.182 \\
\hline
\end{tabular}

Statistical significance by exact logistic regression, unadjusted for within-subject correlations (due to low event counts). No statistically significant differences after adjusting for multiple comparisons (Benjamini-Hochberg, $m=30$ ).

patients with coexisting deformity, whereby removal of bony elements and soft-tissue disruption have the potential to exacerbate existing structural instability and cause recurrence of symptoms.

Kelleher et al. conducted a retrospective review of 75 patients undergoing tubular MIS-ULBD for focal spinal stenosis with or without coexisting scoliosis (mean Cobb angle $14^{\circ}$ ) with a mean 47.5 -month follow-up..$^{38}$ The authors reported significant improvements in ODI scores in patients with deformity with no significant progression of scoliosis; however, there was a $25 \%$ reoperation rate, with $50 \%$ of these failures in patients with concurrent lateral listhesis. Conversely, a recent 2017 prospective study with 2-year follow-up compared outcomes in 207 patients with spinal stenosis with or without coexisting scoliosis (mean Cobb angle $14^{\circ}$ ) following tubular MIS-ULBD ${ }^{37}$ and found significant increases in functional outcomes in all patients with an $8 \%$ reoperation rate, which was associated with preoperative scoliotic disc wedging (Cobb angle $\geq 3^{\circ}$ ) and lateral listhesis. Our tubular MIS-ULBD cohort was found to have a reoperation rate of $11 \%$, which is consistent with rates reported in the literature. Interestingly, no patients in our endoscopic cohort underwent reoperation within the 1-year follow-up period.

To our knowledge, there are limited data regarding the use of decompressive endoscopic spine techniques to treat lumbar spinal stenosis in the setting of deformity and spondylolisthesis. Telfeian et al. reported a series of 4 patients who underwent transforaminal endoscopic decompression to treat unilateral radiculopathy from a disc herniation in the setting of deformity with lateral listhesis. ${ }^{51}$ Madhavan et al. conducted a retrospective analysis of 16 patients with moderate coronal deformity (mean Cobb angle $16.8^{\circ}$ ) who underwent endoscopic transforaminal decompression for unilateral radiculopathy with a mean 7.5-month followup..$^{42}$ All patients had significant improvement in patient reported outcome scores, with only 1 patient requiring reoperation at 1 week for reherniation.

Our study represents the first and largest series investigating interlaminar endoscopic decompression in pa- tients with LSS in the setting of degenerative scoliosis and spondylolisthesis. Our results show that endoscopic decompression is a safe and effective alternative for this patient cohort, as there were significant improvements in all patient-reported outcome measures with a minimum of complications reported at 1 year. Patients in the endoscopic cohort achieved MCID for leg pain VAS score in 95\% of cases compared with $82 \%$ of patients achieving MCID in the MIS-ULBD cohort, which, while not significantly different, indicates likely equipoise across techniques. Furthermore, while complication rates were very low in both cohorts, we feel that endoscopic decompression has a more favorable risk profile than MIS-ULBD with fewer overall adverse events and a lower reoperation rate. Most adverse events experienced in the MIS cohort consisted of urinary retention, which, while a relatively minor complication, can contribute to increased hospital length of stay and should be considered meaningful. Endoscopic decompression also results in significantly faster recovery, reflected by a shorter hospital length of stay and more rapid improvement in ODI during early follow-up.

Our univariate analysis was not able to identify any preoperative factors to predict poor outcome or reoperation. Previous studies, however, postulated that preoperative scoliotic disc wedging (Cobb angle $\geq 3^{\circ}$ ) and lateral listhesis $s^{37,38}$ are associated with reoperation, while spur formation on the concave side of scoliotic curves may be a protective factor in curve progression. ${ }^{35}$ Blumenthal et al. reported that in patients with degenerative grade I lumbar spondylolisthesis who underwent conventional laminectomy, a facet angle $>50^{\circ}$ was associated with a $39 \%$ rate of reoperation, and a disc height $>6.5 \mathrm{~mm}$ was associated with a $45 \%$ rate of reoperation. ${ }^{10}$ While our patient population demonstrated a trend toward worse outcomes with increasing disc height, Cobb angle, and facet angle measurements, there were no statistically significant radiographic parameters that predicted poor outcomes after adjusting for multiple comparisons.

Although sagittal spinal parameters are critical elements in optimizing outcomes, the inability to restore 
sagittal alignment in patients undergoing decompression for spinal stenosis in the setting of degenerative scoliosis (without major instability) has not been shown to have an impact on outcomes. ${ }^{5}$ Our patient cohort represents patients with mild to moderate coronal deformity (mean Cobb angle $15.9^{\circ}$ ) and minimal sagittal imbalance (PI-LL mismatch $\left.=15 \cdot 6^{\circ}\right)$. Patients with clinically significant deformity and sagittal imbalance are unlikely to benefit from these minimally invasive techniques.

While we present evidence that full-endoscopic decompression is a viable alternative to current treatment options, our report has several limitations. This study is a retrospective review of prospectively collected data in a relatively small patient cohort with 1-year follow-up. Our patient selection was not randomized but rather reflects an evolution in practice, which may introduce selection bias. Patients who underwent tubular MIS decompression in our study achieved VAS leg pain score reductions $(-4.1)$, which is comparable with those published in the literature at $\geq 1$-year follow-up $(-4.6){ }^{3}$ The reduction in ODI score in our MIS group was 24.5, with $86 \%$ of these patients achieving an MCID in ODI score. This is comparable to previously reported outcomes after MIS-ULBD (ODI score reduction: 16.4; proportion of MCID: $54.8 \%{ }^{3}$ ). Therefore, we are confident that our tubular MIS decompression outcomes represent an appropriate reference for comparison to the endoscopic decompression cohort.

We anticipate that with longer follow-up, additional patients in the endoscopic group may require reoperation due to the degenerative cascade. We have not routinely performed imaging in patients without new symptomatology and are thus unable to report the radiographic follow-up for our study group; however, we emphasize that clinical outcomes and not radiographic measurements were used as the basis by which to draw conclusions. Our results may not apply to patients who suffer from symptomatic imbalance with progressive deformity and inability to stand or walk. These patients were excluded from the present study, which might lead to a selection bias; however, the focus of this analysis was on patients whose predominant complaints are related to lumbar spinal stenosis in the setting of mild to moderate deformity.

\section{Conclusions}

Full-endoscopic decompression represents a promising treatment option for patients with lumbar spinal stenosis and concurrent mild to moderate degenerative scoliosis and spondylolisthesis. While the decision to perform a decompression, short-segment, or long-segment fusion will ultimately depend on a variety of patient factors and surgeon preference, the endoscopic approach offers an effective option with a favorable risk profile in the appropriately selected patient. Future research is needed to determine the long-term benefits and cost-effectiveness of endoscopic decompression when treating this particular patient cohort.

\section{References}

1. Aebi M: The adult scoliosis. Eur Spine J 14:925-948, 2005

2. Ahn Y: Percutaneous endoscopic decompression for lumbar spinal stenosis. Expert Rev Med Devices 11:605-616, 2014
3. Alimi M, Hofstetter CP, Pyo SY, Paulo D, Härtl R: Minimally invasive laminectomy for lumbar spinal stenosis in patients with and without preoperative spondylolisthesis: clinical outcome and reoperation rates. J Neurosurg Spine 22:339-352, 2015

4. Bae HW, Rajaee SS, Kanim LE: Nationwide trends in the surgical management of lumbar spinal stenosis. Spine (Phila Pa 1976) 38:916-926, 2013

5. Bayerl SH, Pöhlmann F, Finger T, Onken J, Franke J, Czabanka M, et al: The sagittal balance does not influence the 1 year clinical outcome of patients with lumbar spinal stenosis without obvious instability after microsurgical decompression. Spine (Phila Pa 1976) 40:1014-1021, 2015

6. Benner B, Ehni G: Degenerative lumbar scoliosis. Spine (Phila Pa 1976) 4:548-552, 1979

7. Berven SH, Deviren V, Mitchell B, Wahba G, Hu SS, Bradford DS: Operative management of degenerative scoliosis: an evidence-based approach to surgical strategies based on clinical and radiographic outcomes. Neurosurg Clin N Am 18:261-272, 2007

8. Beurskens AJ, de Vet HC, Köke AJ, van der Heijden GJ, Knipschild PG: Measuring the functional status of patients with low back pain. Assessment of the quality of four disease-specific questionnaires. Spine (Phila Pa 1976) 20:10171028,1995

9. Birjandian Z, Emerson S, Telfeian AE, Hofstetter CP: Interlaminar endoscopic lateral recess decompression-surgical technique and early clinical results. J Spine Surg 3:123-132, 2017

10. Blumenthal C, Curran J, Benzel EC, Potter R, Magge SN, Harrington JF Jr, et al: Radiographic predictors of delayed instability following decompression without fusion for degenerative grade I lumbar spondylolisthesis. J Neurosurg Spine 18:340-346, 2013

11. Boukebir MA, Berlin CD, Navarro-Ramirez R, Heiland T, Schöller K, Rawanduzy C, et al: Ten-step minimally invasive spine lumbar decompression and dural repair through tubular retractors. Oper Neurosurg (Hagerstown) 13:232-245, 2017

12. Brodke DS, Annis P, Lawrence BD, Woodbury AM, Daubs MD: Reoperation and revision rates of 3 surgical treatment methods for lumbar stenosis associated with degenerative scoliosis and spondylolisthesis. Spine (Phila Pa 1976) 38:2287-2294, 2013

13. Deyo RA: Treatment of lumbar spinal stenosis: a balancing act. Spine J 10:625-627, 2010

14. Deyo RA, Martin BI, Ching A, Tosteson AN, Jarvik JG, Kreuter W, et al: Interspinous spacers compared with decompression or fusion for lumbar stenosis: complications and repeat operations in the Medicare population. Spine (Phila Pa 1976) 38:865-872, 2013

15. Deyo RA, Mirza SK, Martin BI: Back pain prevalence and visit rates: estimates from U.S. national surveys, 2002. Spine (Phila Pa 1976) 31:2724-2727, 2006

16. Deyo RA, Mirza SK, Martin BI, Kreuter W, Goodman DC, Jarvik JG: Trends, major medical complications, and charges associated with surgery for lumbar spinal stenosis in older adults. JAMA 303:1259-1265, 2010

17. Epstein JA, Epstein BS, Jones MD: Symptomatic lumbar scoliosis with degenerative changes in the elderly. Spine (Phila Pa 1976) 4:542-547, 1979

18. Etminan M, Girardi FP, Khan SN, Cammisa FP Jr: Revision strategies for lumbar pseudarthrosis. Orthop Clin North Am 33:381-392, 2002

19. Fairbank JC, Pynsent PB: The Oswestry Disability Index. Spine (Phila Pa 1976) 25:2940-2952, 2000

20. Farrar JT, Portenoy RK, Berlin JA, Kinman JL, Strom BL: Defining the clinically important difference in pain outcome measures. Pain 88:287-294, 2000 
21. Farrar JT, Young JP Jr, LaMoreaux L, Werth JL, Poole RM: Clinical importance of changes in chronic pain intensity measured on an 11-point numerical pain rating scale. Pain 94:149-158, 2001

22. Foley KT, Smith MM, Rampersaud YR: Microendoscopic approach to far-lateral lumbar disc herniation. Neurosurg Focus 7(5):e5, 1999

23. Fontes RB, Fessler RG: Lumbar radiculopathy in the setting of degenerative scoliosis: MIS decompression and limited correction are better options. Neurosurg Clin N Am 28:335-339, 2017

24. Frazier DD, Lipson SJ, Fossel AH, Katz JN: Associations between spinal deformity and outcomes after decompression for spinal stenosis. Spine (Phila Pa 1976) 22:2025-2029, 1997

25. Fritzell $P$, Hägg $O$, Nordwall A: Complications in lumbar fusion surgery for chronic low back pain: comparison of three surgical techniques used in a prospective randomized study. A report from the Swedish Lumbar Spine Study Group. Eur Spine J 12:178-189, 2003

26. Ghogawala Z, Dziura J, Butler WE, Dai F, Terrin N, Magge $\mathrm{SN}$, et al: Laminectomy plus fusion versus laminectomy alone for lumbar spondylolisthesis. N Engl J Med 374:14241434,2016

27. Gibson JNA, Subramanian AS, Scott CEH: A randomised controlled trial of transforaminal endoscopic discectomy vs microdiscectomy. Eur Spine J 26:847-856, 2017

28. Guha D, Heary RF, Shamji MF: Iatrogenic spondylolisthesis following laminectomy for degenerative lumbar stenosis: systematic review and current concepts. Neurosurg Focus 39(4):E9, 2015

29. Hägg O, Fritzell P, Ekselius L, Nordwall A: Predictors of outcome in fusion surgery for chronic low back pain. A report from the Swedish Lumbar Spine Study. Eur Spine J 12:22-33, 2003

30. Hamasaki T, Tanaka N, Kim J, Okada M, Ochi M, Hutton WC: Biomechanical assessment of minimally invasive decompression for lumbar spinal canal stenosis: a cadaver study. J Spinal Disord Tech 22:486-491, 2009

31. Hasegawa K, Kitahara K, Shimoda H, Hara T: Biomechanical evaluation of destabilization following minimally invasive decompression for lumbar spinal canal stenosis. J Neurosurg Spine 18:504-510, 2013

32. Healey JH, Lane JM: Structural scoliosis in osteoporotic women. Clin Orthop Relat Res (195):216-223, 1985

33. Herkowitz HN, Kurz LT: Degenerative lumbar spondylolisthesis with spinal stenosis. A prospective study comparing decompression with decompression and intertransverse process arthrodesis. J Bone Joint Surg Am 73:802-808, 1991

34. Hoogland T, Schubert M, Miklitz B, Ramirez A: Transforaminal posterolateral endoscopic discectomy with or without the combination of a low-dose chymopapain: a prospective randomized study in 280 consecutive cases. Spine (Phila Pa 1976) 31:E890-E897, 2006

35. Hosogane N, Watanabe K, Kono H, Saito M, Toyama Y, Matsumoto M: Curve progression after decompression surgery in patients with mild degenerative scoliosis. J Neurosurg Spine 18:321-326, 2013

36. Houten JK, Nasser R: Symptomatic progression of degenerative scoliosis after decompression and limited fusion surgery for lumbar spinal stenosis. J Clin Neurosci 20:613-615, 2013

37. Kato M, Namikawa T, Matsumura A, Konishi S, Nakamura $\mathrm{H}$ : Radiographic risk factors of reoperation following minimally invasive decompression for lumbar canal stenosis associated with degenerative scoliosis and spondylolisthesis. Global Spine J 7:498-505, 2017

38. Kelleher MO, Timlin M, Persaud O, Rampersaud YR: Suc- cess and failure of minimally invasive decompression for focal lumbar spinal stenosis in patients with and without deformity. Spine (Phila Pa 1976) 35:E981-E987, 2010

39. Kepler CK, Vaccaro AR, Hilibrand AS, Anderson DG, Rihn JA, Albert TJ, et al: National trends in the use of fusion techniques to treat degenerative spondylolisthesis. Spine (Phila Pa 1976) 39:1584-1589, 2014

40. Komp M, Hahn P, Oezdemir S, Giannakopoulos A, Heikenfeld R, Kasch R, et al: Bilateral spinal decompression of lumbar central stenosis with the full-endoscopic interlaminar versus microsurgical laminotomy technique: a prospective, randomized, controlled study. Pain Physician 18:61-70, 2015

41. Lee CH, Choi M, Ryu DS, Choi I, Kim CH, Kim HS, et al: Efficacy and safety of full-endoscopic decompression via interlaminar approach for central or lateral recess spinal stenosis of the lumbar spine: a meta-analysis. Spine (Phila Pa 1976) 43:1756-1764, 2018

42 Madhavan K, Chieng LO, McGrath L, Hofstetter CP, Wang MY: Early experience with endoscopic foraminotomy in patients with moderate degenerative deformity. Neurosurg Focus 40(2):E6, 2016

43. Murata Y, Takahashi K, Hanaoka E, Utsumi T, Yamagata M, Moriya H: Changes in scoliotic curvature and lordotic angle during the early phase of degenerative lumbar scoliosis. Spine (Phila Pa 1976) 27:2268-2273, 2002

44. Park P, Garton HJ, Gala VC, Hoff JT, McGillicuddy JE: Adjacent segment disease after lumbar or lumbosacral fusion: review of the literature. Spine (Phila Pa 1976) 29:19381944,2004

45. Ruetten S, Komp M, Merk H, Godolias G: Surgical treatment for lumbar lateral recess stenosis with the full-endoscopic interlaminar approach versus conventional microsurgical technique: a prospective, randomized, controlled study. J Neurosurg Spine 10:476-485, 2009

46. Sairyo K, Goel VK, Masuda A, Biyani A, Ebraheim N, Mishiro T, et al: Biomechanical rationale of endoscopic decompression for lumbar spondylolysis as an effective minimally invasive procedure - a study based on the finite element analysis. Minim Invasive Neurosurg 48:119-122, 2005

47. Siepe CJ, Sauer D, Michael Mayer H: Full endoscopic, bilateral over-the-top decompression for lumbar spinal stenosis. Eur Spine J 27 (Suppl 4):563-565, 2018

48. Simmons ED: Surgical treatment of patients with lumbar spinal stenosis with associated scoliosis. Clin Orthop Relat Res (384): 45-53, 2001

49. Tacconi L, Baldo S, Merci G, Serra G: Transforaminal percutaneous endoscopic lumbar discectomy: outcome and complications in 270 cases. J Neurosurg Sci [epub ahead of print], 2018

50. Taylor WR, Chen JW, Meltzer H, Gennarelli TA, Kelbch C, Knowlton S, et al: Quantitative pupillometry, a new technology: normative data and preliminary observations in patients with acute head injury. Technical note. J Neurosurg 98:205213, 2003

51. Telfeian AE, Oyelese A, Fridley J, Gokaslan ZL: Transforaminal endoscopic decompression in the setting of lateral lumbar spondylolisthesis. World Neurosurg 117:321-325, 2018

52. Thomé C, Zevgaridis D, Leheta O, Bäzner H, Pöckler-Schöniger C, Wöhrle J, et al: Outcome after less-invasive decompression of lumbar spinal stenosis: a randomized comparison of unilateral laminotomy, bilateral laminotomy, and laminectomy. J Neurosurg Spine 3:129-141, 2005

53. Transfeldt EE, Topp R, Mehbod AA, Winter RB: Surgical outcomes of decompression, decompression with limited fusion, and decompression with full curve fusion for degenerative scoliosis with radiculopathy. Spine (Phila Pa 1976) 35:1872-1875, 2010 
54. Weinstein JN, Tosteson TD, Lurie JD, Tosteson A, Blood E, Herkowitz H, et al: Surgical versus nonoperative treatment for lumbar spinal stenosis four-year results of the Spine Patient Outcomes Research Trial. Spine (Phila Pa 1976) 35:1329-1338, 2010

55. Zanirato A, Damilano M, Formica M, Piazzolla A, Lovi A, Villafañe JH, et al: Complications in adult spine deformity surgery: a systematic review of the recent literature with reporting of aggregated incidences. Eur Spine J 27:22722284,2018

56. Zhang B, Liu S, Liu J, Yu B, Guo W, Li Y, et al: Transforaminal endoscopic discectomy versus conventional microdiscectomy for lumbar discherniation: a systematic review and meta-analysis. J Orthop Surg Res 13:169, 2018

\section{Disclosures}

Dr. Hofstetter: consultant for Johnson \& Johnson, Globus, and Joimax.

\section{Author Contributions}

Conception and design: Hofstetter, Hasan, McGrath, Sen. Acquisition of data: Hofstetter, Hasan, McGrath, Sen. Analysis and interpretation of data: Hasan, McGrath, Sen, Barber. Drafting the article: Hofstetter, McGrath, Sen. Critically revising the article: Hofstetter, McGrath. Reviewed submitted version of manuscript: Hofstetter, McGrath. Statistical analysis: McGrath, Barber.

\section{Correspondence}

Christoph Hofstetter: University of Washington, Seattle, WA. chh9045@uw.edu. 